\title{
RESTRICTED CONVOLUTION INEQUALITIES, MULTILINEAR OPERATORS AND APPLICATIONS
}

\author{
Dan-Andrei Geba, Allan Greenleaf, Alex Iosevich, \\ EyVindur Palsson AND ERIC SAWYER
}

Abstract. For functions $F, G$ on $\mathbb{R}^{n}$, any $k$-dimensional affine subspace $H \subset \mathbb{R}^{n}, 1 \leq$ $k<n$, and exponents $p, q, r \geq 2$ with $\frac{1}{p}+\frac{1}{q}+\frac{1}{r}=1$, we prove the estimate

$$
\left\|\left.(F * G)\right|_{H}\right\|_{L^{r}(H)} \leq\|F\|_{\Lambda_{2, p}^{H}\left(\mathbb{R}^{n}\right)} \cdot\|G\|_{\Lambda_{2, q}^{H}\left(\mathbb{R}^{n}\right)} .
$$

Here, the mixed norms on the right are defined in terms of the Fourier transform by

$$
\|F\|_{\Lambda_{2, p}^{H}\left(\mathbb{R}^{n}\right)}=\left(\int_{H^{*}}\left(\int|\widehat{F}|^{2} d H_{\xi}^{\perp}\right)^{\frac{p}{2}} d \xi\right)^{1 / p},
$$

with $d H_{\xi}^{\perp}$ the $(n-k)$-dimensional Lebesgue measure on the affine subspace $H_{\xi}^{\perp}:=$ $\xi+H^{\perp}$. Dually, one obtains restriction theorems for the Fourier transform on affine subspaces. We use this, and a maximal variant, to prove results for a variety of multilinear convolution operators, including $L^{p}$-improving bounds for measures; bilinear variants of Stein's spherical maximal theorem; estimates for $m$-linear oscillatory integral operators; Sobolev trace inequalities; and bilinear estimates for solutions to the wave equation.

\section{CONTEnts}

1. Introduction 676

2. Proofs of restricted convolution and maximal estimates 680

2.1. Proof of Theorem $1.3 \quad 680$

2.2. Proof of Theorem $1.8 \quad 680$

3. $L^{p}$ improving and maximal estimates for multilinear operator $\quad 682$

$\begin{array}{lll}3.1 . & L^{p} \text {-improving measures } & 683\end{array}$

3.2. Proof of Theorem 3.3 684

3.3. Bilinear analogues of Stein's spherical maximal theorem 685

3.4. Proof of Corollary $3.9 \quad 686$

4. Applications to PDE 686

$\begin{array}{ll}\text { 4.1. Sobolev traces on subspaces } & 687\end{array}$

4.2. The heat equation: restriction of solutions to subspaces 688

4.3. The wave equation: restriction to subspaces 689

4.4. The wave equation: product of solutions 690

5. Bilinear oscillatory integral operators 690

6. Further thoughts and open problems $\quad 692$

$\begin{array}{ll}\text { Acknowledgments } & 693\end{array}$

References $\quad 693$

Received by the editors March 4, 2013. 


\section{Introduction}

Convolution inequalities play a central role in harmonic analysis and related areas, with perhaps the most classical of these being Young's inequality: if $F, G \in \mathcal{S}\left(\mathbb{R}^{n}\right)$, then, for $p, q, r \geq 1$,

$$
\|F * G\|_{L^{r}\left(\mathbb{R}^{n}\right)} \leq\|F\|_{L^{p}\left(\mathbb{R}^{n}\right)}\|G\|_{L^{q}\left(\mathbb{R}^{n}\right)}, \quad \frac{1}{r}=\frac{1}{p}+\frac{1}{q}-1 .
$$

Many of the applications of this estimate take the following form. (See, e.g., [25] and references therein.) Let $F(x)=f(x)$, a function, and $g(x)=K(x)$, a suitable kernel or measure. Then (1.1) implies Lebesgue space mapping properties of the linear convolution operator,

$$
T f(x)=\int f(x-y) K(y) d y \text {. }
$$

In this paper, we prove an estimate for restrictions of convolutions on $\mathbb{R}^{n}$ to affine subspaces, and show that this can be applied to obtain multilinear analogues for a range of well-known results in linear harmonic analysis. Consequences include Fourier restriction theorems for linear subspaces; $L^{p}$ improving properties for multilinear generalized Radon transforms and more general convolution operators; and trace theorems and bilinear estimates for solutions of the heat and wave equations. We also obtain a maximal variant of the restricted convolution estimate and use it to to obtain multilinear variants of Stein's spherical maximal theorem [24]. Multilinear operators, especially convolutions, have been widely studied; see, e.g., [19, 12, 13, 30, 16]. We focus here on showing that a variety of apparently quite different multilinear results can be viewed through the unifying framework of restricted convolution.

The papers $[11,15]$ gave bounds for multilinear operators with positive kernels that proved effective in the context of Falconer type problems in geometric measure theory. Unfortunately, the reliance on positivity restricted their applicability to problems where positivity is a natural condition. Other problems in harmonic analysis, such as maximal operators and $L^{p}$-improving properties of measures, typically require methods where positivity is violated. In this paper, we address this issue by developing a mechanism that produces multilinear bounds as a consequence of trace estimates, for which positivity is not a factor.

To state the general result on restrictions of convolutions to affine subspaces, we introduce some notation. For $1 \leq k \leq n$, let $H \subset \mathbb{R}^{n}$ be a $k$-dimensional linear subspace, $i_{H}: H \hookrightarrow \mathbb{R}^{n}$ the inclusion map, and $i_{H}^{*}:\left(R^{n}\right)^{*} \longrightarrow H^{*}$ the dual restriction map. $\left(\mathbb{R}^{n}\right)^{*}$ foliates into a union of $(n-k)$-dimensional affine subspaces, parallel to $\left(i_{H}^{*}\right)^{-1}(0)=H^{\perp}$

$$
\left(\mathbb{R}^{n}\right)^{*}=\bigcup_{\xi \in H^{*}}\left(i_{H}^{*}\right)^{-1}(\xi)
$$

Via the Euclidean metric, one identifies $\left(i_{H}^{*}\right)^{-1}(\xi)$ with $H_{\xi}^{\perp}:=\xi+H^{\perp}$. The Lebesgue measure $d \zeta$ on $\left(\mathbb{R}^{n}\right)^{*}$ thus decomposes as $d \zeta=d H_{\xi}^{\perp}(\zeta) d \xi$, where $d H_{\xi}^{\perp}$ and $d \xi$ are the Lebesgue measures of dimensions $n-k$ and $k$ on $H_{\xi}^{\perp}$ and $H^{*}$, resp. Relative to this decomposition of the Fourier variables, we define a scale of mixed-norm spaces: 
Definition 1.1. For $1 \leq r, p \leq \infty$ and $F \in \mathcal{S}\left(\mathbb{R}^{n}\right)$, let

$$
\|F\|_{\Lambda_{r, p}^{H}\left(\mathbb{R}^{n}\right)}=\left(\int_{H^{*}}\left[\int_{H_{\xi}^{\perp}}|\widehat{F}(\zeta)|^{r} d H_{\xi}^{\perp}(\zeta)\right]^{\frac{p}{r}} d \xi\right)^{1 / p},
$$

and $\Lambda_{r, p}^{H}:=\Lambda_{r, p}^{H}\left(\mathbb{R}^{n}\right)$ be the completion of $\mathcal{S}\left(\mathbb{R}^{n}\right)$ with respect to this norm.

Remark 1.2. If $k=n$ or $r=p,\|F\|_{\Lambda_{r, p}^{H}}=\|\widehat{F}\|_{L^{p}}$, and if $r=p=2$, this equals $\|F\|_{L^{2}}$. Furthermore, the norm is translation-invariant, so it is natural, if $\tilde{H} \subset \mathbb{R}^{n}$ is an affine $k$-plane, to define $\|\cdot\|_{\Lambda_{r, p}^{\tilde{H}}}$ by using $H$ and $H_{\xi}^{\perp}$ on the right-hand side of (1.3), with $H$ the translate of $\tilde{H}$ passing through 0 .

We can now state our first result.

Theorem 1.3. Let $1 \leq k<n, H \subset \mathbb{R}^{n}$ a $k$-dimensional affine subspace, and $F, G \in$ $\mathcal{S}\left(\mathbb{R}^{n}\right)$. Then, for $\frac{1}{p}+\frac{1}{q}+\frac{1}{r}=1$, with $p, q, r \geq 2$,

$$
\left\|\left.(F * G)\right|_{H}\right\|_{L^{r}(H)} \leq\left\|\left.F\right|_{\Lambda_{2, p}^{H}\left(\mathbb{R}^{n}\right)} \cdot\right\| G \|_{\Lambda_{2, q}^{H}\left(\mathbb{R}^{n}\right)} \cdot
$$

Moreover, the best constant in (1.4), considering $\left.F \rightarrow F * G\right|_{H}$ as a linear operator acting on $F$, is

$$
\|F \longrightarrow F * G\|_{L^{2}\left(\mathbb{R}^{n}\right) \longrightarrow L^{2}(H)}=\|G\|_{\Lambda_{2, \infty}^{H}\left(\mathbb{R}^{n}\right)} .
$$

As will be seen below, Theorem 1.3 is proved by a straightforward $T T^{*}$ argument and interpolation, but it is not obvious in advance that such a result should hold, and it turns out to have an array of interesting multilinear applications. One motivation for considering inequalities such as (1.4) comes from the study of multilinear convolution operators. For $d \geq 1, m \geq 2$, denote elements of $\mathbb{R}^{m d}$ by $\vec{x}:=\left(x^{1}, \ldots, x^{m}\right)$ and elements of $\left(\mathbb{R}^{m d}\right)^{*}$ by $\vec{\xi}:=\left(\xi^{1}, \ldots, \xi^{m}\right){ }^{1}$ For a kernel $K(\vec{x}) \in \mathcal{D}^{\prime}\left(\mathbb{R}^{m d}\right)$, define an $m$-linear convolution operator $T:\left(\mathcal{D}\left(\mathbb{R}^{d}\right)\right)^{m} \rightarrow \mathcal{E}\left(\mathbb{R}^{d}\right)$ by

$$
T\left(f_{1}, \ldots, f_{m}\right)(x)=\int_{\mathbb{R}^{m d}} f_{1}\left(x-u^{1}\right) \cdots f_{m}\left(x-u^{m}\right) K(\vec{u}) d u^{1} \cdots d u^{m}, \quad x \in \mathbb{R}^{d}
$$

Since $T\left(f_{1}, \ldots, f_{m}\right)(x)=\left(\left(f_{1} \otimes \cdots \otimes f_{m}\right) * K\right)(x, \ldots, x)$, multilinear bounds for $T$ can be obtained from the restricted convolution estimate of Theorem 1.3, with $H$ being the diagonal $H_{0}$ of $\mathbb{R}^{m d}, H_{0}:=\left\{(x, \ldots, x): x \in \mathbb{R}^{d}\right\}$. An immediate consequence is:

Corollary 1.4. Let $K \in \mathcal{E}^{\prime}\left(\mathbb{R}^{m d}\right), f_{1}, \ldots, f_{m} \in \mathcal{S}\left(\mathbb{R}^{d}\right)$, and $2 \leq r \leq \infty$. Then, the multilinear convolution operator defined by (1.6) satisfies

$$
\left\|T\left(f_{1}, \ldots, f_{m}\right)\right\|_{L^{r}\left(\mathbb{R}^{d}\right)} \lesssim\|K\|_{\Lambda_{2, \frac{2 r}{r-2}}^{H_{0}}} \cdot \prod_{j=1}^{m}\left\|f_{j}\right\|_{L^{2}\left(\mathbb{R}^{d}\right)} .
$$

In Section 3, we show how Corollary 1.4 can applied to obtain $L^{p}$-improving results for multilinear convolutions with measures, natural analogues of those in the linear setting.

\footnotetext{
${ }^{1}$ The notation $\vec{x}, \vec{\xi}$, etc., will occasionally also be used for $l$-tuples of vectors in $\mathbb{R}^{d}$ for $l<m$.
} 
Note that $H_{0}^{*}$ can be identified with $\left\{\left(\frac{\xi}{m}, \ldots, \frac{\xi}{m}\right): \xi \in\left(\mathbb{R}^{d}\right)^{*}\right\}$, while on the antidiagonal, $H_{0}^{\perp}=\left\{\vec{\eta}=\left(\eta^{1}, \ldots, \eta^{m}\right): \sum \eta^{j}=0\right\}$, we can solve for $\eta^{m}$ in terms of $\left(\eta^{1}, \ldots, \eta^{m-1}\right)$. This give rise to a parametrization making concrete the decomposition (1.2),

$\rho_{H_{0}}: \mathbb{R}_{\xi}^{d} \times \mathbb{R}_{\vec{\eta}}^{(m-1) d} \rightarrow\left(\mathbb{R}^{m d}\right)^{*}, \quad \rho_{H}(\xi, \vec{\eta})=\left(\frac{\xi}{m}+\eta^{1}, \ldots, \frac{\xi}{m}+\eta^{m-1}, \frac{\xi}{m}-\sum_{j=1}^{m-1} \eta^{j}\right)$.

(In the bilinear case, $m=2$, it is convenient to use the more symmetric parametrization of $\rho_{H_{0}}(\xi, \eta)=\left(\frac{\xi-\eta}{2}, \frac{\xi+\eta}{2}\right)$.) One can then express the norm of $K$ in (1.7) as

$$
\|K\|_{\Lambda_{2, \frac{2 r}{r-2}}^{H_{0}}}=c\left[\int_{\mathbb{R}^{d}}\left(\int_{\mathbb{R}^{(m-1) d}}\left|\widehat{K}\left(\rho_{H_{0}}(\xi, \vec{\eta})\right)\right|^{2} d \vec{\eta}\right)^{\frac{r}{r-2}} d \xi\right]^{r-2 / 2 r} .
$$

Another type of application comes from noting that, upon application of the Fourier transform, Theorem 1.3 becomes a bilinear Fourier restriction estimate. Interchanging the roles of $F, G$ and $\widehat{F}, \widehat{G}$, consider $H$ as a subspace of $\left(\mathbb{R}^{n}\right)^{*}$ and, as with the diagonal $H_{0} \subset \mathbb{R}^{m d}$ above, introduce linear coordinates $u \in \mathbb{R}^{k}, v \in \mathbb{R}^{n-k}$ on $H^{*}, H^{\perp}$. This gives rise to a linear isomorphism $\rho_{H}: \mathbb{R}_{u}^{k} \times \mathbb{R}_{v}^{n-k} \rightarrow \mathbb{R}^{n}$. Theorem 1.3 then yields:

Corollary 1.5. Let $H \subset\left(\mathbb{R}^{n}\right)^{*}$ be a $k$-dimensional subspace, $0 \leq k \leq n$, and $F, G \in$ $\mathcal{S}\left(\mathbb{R}^{n}\right)$. Then, for $p, q, r \geq 2, \frac{1}{p}+\frac{1}{q}+\frac{1}{r}=1$, we have

$$
\left\|\left.\widehat{F G}\right|_{H}\right\|_{L^{r}(H)} \lesssim\left\|F \circ \rho_{H}\right\|_{L_{u}^{p} L_{v}^{2}} \cdot\left\|G \circ \rho_{H}\right\|_{L_{u}^{q} L_{v}^{2}},
$$

where $L_{u}^{p} L_{v}^{2}$ denotes the mixed-norm space with norm $\left(\int_{\mathbb{R}^{k}}\left(\int_{\mathbb{R}^{n-k}}|F(u, v)|^{2} d v\right)^{\frac{p}{2}} d u\right)^{\frac{1}{p}}$.

Remark 1.6. The extreme cases of $\operatorname{dim}(H)=n$ or 0 in Corollary 1.5 correspond to standard facts: For $H=\mathbb{R}^{n}$, one has $H^{\perp}=(0)$ and $L_{u}^{p} L_{v}^{2}=L_{u}^{p}$, so that $F G \in L^{r^{\prime}}$ by Hölder and then $\widehat{F G} \in L^{r}$ by Hausdorff-Young. On the other hand, for $H=(0), H^{\perp}=\mathbb{R}^{n}, L_{u}^{p} L_{v}^{2}=L_{v}^{2}$ and $F G \in L^{1} \Longrightarrow \widehat{F G}(0)$ is well-defined.

Starting with a single function $F$, replacing $F$ and $G$ in Corollary 1.5 by $|F|^{\frac{1}{2}}$ and $|F|^{\frac{1}{2}} \cdot \frac{F}{|F|}$, resp., convolving each with an approximate identity to restore membership in $\mathcal{S}$, and passing to the limit, one obtains a linear Fourier restriction theorem for linear subspaces:

Corollary 1.7. If $H \subset\left(\mathbb{R}^{n}\right)^{*}$ is a $k$-dimensional subspace and $F \in \mathcal{S}\left(\mathbb{R}^{n}\right)$, then, for $p, q, r \geq 2, \frac{1}{p}+\frac{1}{q}+\frac{1}{r}=1$, one has

$$
\left\|\left.\widehat{F}\right|_{H}\right\|_{L^{r}(H)} \lesssim\left\|\left.F \circ \rho_{H}\right|_{L_{u}^{p} L_{v}^{1}} ^{\frac{1}{2}} \cdot\right\| F \circ \rho_{H} \|_{L_{u}^{q} L_{v}^{1}}^{\frac{1}{2}}, \quad p, q, r \geq 2, \frac{1}{p}+\frac{1}{q}+\frac{1}{r}=1,
$$

and

$$
\left\|\left.\widehat{F}\right|_{H}\right\|_{L^{r}(H)} \lesssim\left\|F \circ \rho_{H}\right\|_{L_{u}^{p} L_{v}^{1}}, \quad r=\frac{p}{p-2}, p \geq 2
$$


Corollary 1.7 shows that the Fourier transform of a function can be restricted to a linear subspace, provided that the mixed norm is finite; this is in contrast with the well-known fact that the Fourier transforms of general functions in a standard $L^{p}, p>1$, cannot be restricted to subspaces.

Yet another class of applications is based on a maximal operator variant of Theorem 1.3:

Theorem 1.8. Let $F, G \in \mathcal{S}\left(\mathbb{R}^{n}\right)$ and, for $t>0$, define $G_{t}(\vec{x})=t^{-n} G\left(\frac{\vec{x}}{t}\right)$. Let $H \subset \mathbb{R}^{n}$ be a $k$-dimensional subspace. Suppose that, for some $\gamma>\frac{1}{2}$ and any $t>0$,

$$
\left\|G_{t}^{j}\right\|_{\Lambda_{2, \infty}^{H}}:=\sup _{\xi \in H^{*}}\left(\int_{H_{\xi}^{\perp}}\left|\widehat{G}^{j}(t \vec{\zeta})\right|^{2} d H_{\xi}^{\perp}(\vec{\zeta})\right)^{\frac{1}{2}} \lesssim 2^{-j \gamma},
$$

where, for $j \geq 0, G^{j}$ is the Littlewood-Paley component of $G$ at frequency scale $2^{j}$ relative to a dyadic decomposition of $\left(\mathbb{R}^{n}\right)^{*}$, and the same estimate holds for $\nabla \widehat{G}^{j}$. Then,

$$
\left\|\sup _{t>0}\left|F * G_{t}\right|\right\|_{L^{2}(H)} \lesssim\|F\|_{L^{2}\left(\mathbb{R}^{m d}\right)} .
$$

Remark 1.9. Approximating $F$ by a continuous function plus a function with a small $L^{2}$ norm, a standard argument then shows that, for $F \in L^{2}\left(\mathbb{R}^{n}\right)$,

$$
\lim _{t \rightarrow 0} \int_{\mathbb{R}^{n}} F(x-u) G_{t}(u) d u=c_{G} \cdot F(x) \text { a.e., with } c_{G}:=\int_{\mathbb{R}^{n}} G(u) d u .
$$

Returning to the multilinear setting by taking $H=H_{0}, F(\vec{x})=\prod_{j=1}^{m} f_{j}\left(x^{j}\right)$ and $G(\vec{x})=K(\vec{x})$ in Theorem 1.8, we obtain the following bound for maximal $m$-linear convolution operators.

Corollary 1.10. If $K$ is a finite Borel measure on $\mathbb{R}^{m d}, f_{j} \in \mathcal{S}\left(\mathbb{R}^{d}\right), 1 \leq j \leq m$, and $t>0$, define

$$
B_{t}\left(f_{1}, \ldots, f_{m}\right)(x)=\int_{\mathbb{R}^{m d}} f_{1}\left(x-t u^{1}\right) \cdots f_{m}\left(x-t u^{m}\right) d K(\vec{u}),
$$

and the associated maximal operator,

$$
\mathcal{B}\left(f_{1}, \ldots, f_{m}\right)(x)=\sup _{t>0}\left|B_{t}\left(f_{1}, \ldots, f_{m}\right)(x)\right| .
$$

Then

$$
\left\|\mathcal{B}\left(f_{1}, \ldots, f_{m}\right)\right\|_{L^{2}\left(\mathbb{R}^{d}\right)} \lesssim \prod_{j=1}^{m}\left\|f_{j}\right\|_{L^{2}\left(\mathbb{R}^{d}\right)},
$$

provided that $K$ and $\nabla K$ satisfy the Littlewood-Paley decay condition (1.12) w.r.t. $\Lambda_{2, \infty}^{H_{0}}\left(\mathbb{R}^{m d}\right)$.

In Section 3.3 below, we use Corollary 1.10 and some additional machinery to establish $m$-linear analogues of Stein's spherical maximal theorem [24].

We point out that there have been other works on restrictions of convolutions (e.g., $[2,1])$ and estimates for multilinear convolution operators $[16,26]$, but the results there seem to be of a different nature than those obtained here. 
The paper is organized as follows. The proofs of Theorems 1.3 and 1.8 are given in Section 2. In Section 3, we then use Theorems 1.3 and 1.8 to prove estimates for multilinear analogues of both linear $L^{p}$-improving estimates for convolution and Stein's spherical maximal theorem. We show in Section 4 how these results imply certain Sobolev trace inequalities and estimates for solutions of the heat and wave equations. In Section 5, we use our method to derive bounds for multilinear oscillatory integral operators, and Section 6 contains some further discussion and open problems.

\section{Proofs of restricted convolution and maximal estimates}

2.1. Proof of Theorem 1.3. By the translation-invariance of the norms in (1.4), one may assume that $H \subset \mathbb{R}^{n}$ is a $k$-dimensional linear subspace, which, by rotation covariance, can be put in the form $H=\left\{\left(x^{\prime}, x^{\prime \prime}\right) \in \mathbb{R}^{k} \times \mathbb{R}^{n-k} \mid x^{\prime \prime}=0\right\} \sim \mathbb{R}^{k}$, so that, for $\xi^{\prime} \in H^{*}, H_{\xi}^{\perp}=\left\{\left(\xi^{\prime}, \xi^{\prime \prime}\right) \mid \xi^{\prime \prime} \in \mathbb{R}^{n-k}\right\}$. Fixing $G$, define the restricted convolution operator $T F=\left.(F * G)\right|_{H}$, i.e., $T F\left(x^{\prime}\right)=(F * G)\left(x^{\prime}, 0\right)$. Then the formal adjoint is given by

$$
T^{*} h\left(u^{\prime}, u^{\prime \prime}\right)=\int_{\mathbb{R}^{k}} \bar{G}\left(x^{\prime}-u^{\prime},-u^{\prime \prime}\right) h\left(x^{\prime}\right) d x^{\prime},
$$

from which one sees that $T T^{*}$ is a translation-invariant operator on $\mathbb{R}^{k}$, given in Fourier multiplier form by

$$
\widehat{T T^{*} h}\left(\xi^{\prime}\right)=\left(\int_{\mathbb{R}^{n-k}}\left|\widehat{G}\left(\xi^{\prime}, \xi^{\prime \prime}\right)\right|^{2} d \xi^{\prime \prime}\right) \widehat{h}\left(\xi^{\prime}\right)=\left(\int_{H_{\xi}^{\perp}}|\widehat{G}(\vec{\zeta})|^{2} d H_{\xi^{\prime}}^{\perp}(\vec{\zeta})\right) \widehat{h}(\xi) .
$$

By Parseval,

$$
\left\|T T^{*}\right\|_{L^{2}(H) \rightarrow L^{2}(H)}=\sup _{\xi^{\prime} \in H^{*}}\left(\int_{H_{\xi}^{\perp}}|\widehat{G}(\vec{\zeta})|^{2} d H_{\xi^{\prime}}^{\perp}(\vec{\zeta})\right)=\|G\|_{\Lambda_{2, \infty}^{H}}^{2},
$$

and thus $\|T\|_{L^{2}(H) \rightarrow L^{2}\left(\mathbb{R}^{n}\right)}=\|G\|_{\Lambda_{2, \infty}^{H}}$, yielding (1.5), and therefore (1.4) for $p=$ $2, q=\infty, r=2$. On the other hand,

$\|F * G\|_{L^{\infty}(H)} \leq\|F * G\|_{C_{0}\left(\mathbb{R}^{n}\right)} \leq\|\hat{F} \hat{G}\|_{L^{1}\left(\mathbb{R}^{n}\right)} \leq\|\hat{F}\|_{L^{2}} \cdot\|\hat{G}\|_{L^{2}} \leq\|F\|_{\Lambda_{2,2}^{H}} \cdot\|G\|_{\Lambda_{2,2}^{H}}$, which is (1.4) for $p=q=2, r=\infty$. Interpolation then gives (1.4) for $q=2, \frac{1}{p}+\frac{1}{r}=$ $1, p, r \geq 2$. Symmetry in $F, G$ implies that (1.4) also holds for $p=2, \frac{1}{q}+\frac{1}{r}=1, q, r \geq$ 2 ; interpolation then yields (1.4) for general exponents.

2.2. Proof of Theorem 1.8. It suffices to establish the estimate

$$
\left\|\sup _{R} \sup _{R \leq t \leq 2 R}\left|F * G_{t}\right|\right\|_{L^{2}(H)}^{2} \leq\|F\|_{2}^{2}
$$

where $R$ ranges over all dyadic numbers. For fixed $R$, make a Littlewood-Paley decomposition with respect to that scale. First define

$$
\widehat{F^{j}}(\vec{\xi})=\psi\left(2^{-j}|\vec{\xi}|\right) \widehat{F}(\vec{\xi}),
$$

where $\psi$ is a smooth cut-off function supported in the annulus in $\mathbb{R}^{m d}$ of inner radius $\frac{1}{2}$ and outer radius 4 , identically equal to 1 in the annulus of inner radius 1 and 
outer radius 2 , and satisfying $\sum_{j} \psi\left(2^{-j} \cdot\right) \equiv 1$. Letting [.] denote the greatest integer function, we now aim to estimate

$$
\sup _{R \leq t \leq 2 R}\left|F^{j+\left[\log _{2}(R)\right]} * G_{t}\right| .
$$

We shall need the following elementary observation.

Lemma 2.1. Let $F$ be a differentiable function on $[R, 2 R]$. Then

$$
\sup _{t \in[R, 2 R]}|F(t)|^{2} \leq|F(R)|^{2}+2\left(\int_{R}^{2 R}|F(t)|^{2} d t\right)^{\frac{1}{2}} \cdot\left(\int_{R}^{2 R}\left|F^{\prime}(t)\right|^{2} d t\right)^{1 / 2} .
$$

To prove the lemma, just observe that by the fundamental theorem of calculus,

$$
F(t)^{2}=F(R)^{2}+2 \int_{R}^{t} 2 F(s) F^{\prime}(s) d s
$$

and apply the Cauchy-Schwarz inequality. In order to use Lemma 2.1, observe that

$$
\begin{aligned}
\frac{d}{d t}\left(F * G_{t}\right)(x) & =\int e^{2 \pi i x \cdot\left(\xi^{1}+\cdots+\xi^{m}\right)} \widehat{F}(\vec{\xi}) \frac{d}{d t} \widehat{G}(t \vec{\xi}) d \vec{\xi} \\
& =\int e^{2 \pi i x \cdot\left(\xi^{1}+\cdots+\xi^{m}\right)} \widehat{F}(\vec{\xi})\langle\nabla \widehat{G}(t \vec{\xi}), \vec{\xi}\rangle d \vec{\xi} \\
& =\int e^{2 \pi i x \cdot\left(\xi^{1}+\cdots+\xi^{m}\right)} \widehat{F}(\vec{\xi}) t^{-1} \widehat{G^{*}}(t \vec{\xi}) d \vec{\xi}
\end{aligned}
$$

where

Note that

$$
\left|\widehat{G^{*}}(\vec{\xi})\right| \lesssim|\vec{\xi}| \cdot|\nabla \widehat{G}(\vec{\xi})|
$$

$$
\begin{aligned}
\left(F^{j+\left[\log _{2}(R)\right]} * G_{t}\right)(x) & =\int e^{2 \pi i x \cdot\left(\xi^{1}+\cdots+\xi^{m}\right)} \widehat{F}(\vec{\xi}) \psi\left(2^{-j} R|\vec{\xi}|\right) \widehat{G}(t \vec{\xi}) d \vec{\xi} \\
& =\int e^{2 \pi i x \cdot\left(\xi^{1}+\cdots+\xi^{m}\right)} \widehat{F}(\vec{\xi}) \psi\left(2^{-j} t|\vec{\xi}|\right) \widehat{G}(t \vec{\xi}) d \vec{\xi} \\
& =\left(F * G_{t}^{j}\right)(x)
\end{aligned}
$$

when $R \leq t \leq 2 R$, where $G_{t}^{j}$ is given by $\widehat{G_{t}^{j}}(\vec{\xi})=\psi\left(2^{-j} t|\xi|\right) \widehat{G}(t \vec{\xi})$. Similar calculations hold for $G^{*}$.

Applying Lemma 2.1, we see that

$$
\begin{aligned}
\left\|\sup _{t \in[R, 2 R]}\left|F^{j+\left[\log _{2}(R)\right]} * G_{t}\right|_{H} \mid\right\|_{L^{2}(H)}^{2} \leq & \left.|| F * G_{R}^{j}\right|_{H} \|_{L^{2}(H)}^{2} \\
& +2\left(\frac{1}{R} \int_{R}^{2 R} \int\left|\left(F * G_{t}^{j}\right)(x)\right|^{2} d x d t\right)^{1 / 2} \\
& \times\left(R \int_{R}^{2 R} t^{-2} \int\left|\left(F *\left(G^{*}\right)_{t}^{j}\right)(x)\right|^{2} d x d t\right)^{1 / 2} .
\end{aligned}
$$

Now appealing to Theorem 1.3 and using estimate (1.12), we see that this expression is

$$
\lesssim\|F\|_{2}^{2} \cdot 2^{-j(1+2 \epsilon)}+\|F\|_{2}^{2} \cdot 2^{-j\left(\frac{1}{2}+\epsilon\right)} \cdot 2^{-j\left(\frac{1}{2}+\epsilon\right)} 2^{j} .
$$


We could have made the argument stronger, replacing the $\psi$ factor with $\psi\left(2^{-j} R|\xi|\right) \tilde{\psi}\left(2^{-j} R|\xi|\right)$, where $\tilde{\psi}$ is a slight widening of $\psi$. The argument as above would then yield

$$
\left\|\sup _{t \in[R, 2 R]}\left|F^{j+\left[\log _{2}(R)\right]} * G_{t}\right|_{H}\right\|_{L^{2}(H)}^{2} \lesssim 2^{-j \epsilon}\left\|F^{j+\left[\log _{2}(R)\right]}\right\|_{2}^{2},
$$

where the Littlewood-Paley decomposition on the right-hand side is made with respect to the dilates of $\tilde{\psi}$. Summing the corresponding geometric series and bounding a supremum by a square function, we obtain equation (2.1) and thus the conclusion of Theorem 1.8.

\section{3. $L^{p}$ improving and maximal estimates for multilinear operator}

Multilinear operators play a key role in many aspects of harmonic analysis and partial differential equations (PDE). See, for example, [25] and references therein for a comprehensive description and history of this subject. The starting point for us is the work of Coifman and Meyer [8], who initiated the comprehensive study of CalderónZygmund theory in the multilinear setting. They showed that an $m$-linear multiplier operator, acting on a tensor product $f_{1} \otimes \cdots \otimes f_{m}$, maps

$$
M: L^{p_{1}}\left(\mathbb{R}^{d}\right) \times \cdots \times L^{p_{m}}\left(\mathbb{R}^{d}\right) \rightarrow L_{s}^{r}\left(\mathbb{R}^{d}\right)
$$

if

$$
\frac{1}{p_{1}}+\cdots+\frac{1}{p_{m}}=\frac{1}{r}
$$

and the multiplier $m(\vec{\xi})$ of $M$ satisfies

$$
\left|D^{\alpha} m\left(\xi^{1}, \ldots, \xi^{m}\right)\right| \leq C_{\alpha}\left(1+\left|\xi^{1}\right|+\cdots+\left|\xi^{m}\right|\right)^{-s-|\alpha|}, \forall \alpha \in \mathbb{Z}_{+}^{m d} .
$$

This estimate has numerous important applications. However, in many multilinear situations the symbolic decay conditions on the multiplier and its derivatives are violated. For example, if the multiplier is the Fourier transform of the Lebesgue measure on a smooth hypersurface, the derivatives satisfy the same decay estimates as the multiplier, and no better. Another example that is not covered by this result is the case of multilinear fractional integration, handled in [19] using different methods; see also [12].

A systematic study of multilinear analogues of linear generalized Radon transforms was begun in [11], focusing on applications to the study of finite point configurations problems in geometric measure theory. These problems have their roots in the work of Falconer on the distance problem in the continuous setting and of the Erdös school on finite point configurations in the discrete setting; see, e.g., [5] and references therein. The main bilinear estimate in [11] can be stated as follows.

Theorem 3.1. Let $K$ be a non-negative integrable function on $\mathbb{R}^{2 d}$, and define

$$
B(f, g)(x)=\int f(x-u) g(x-v) K(u, v) d u d v .
$$

Then for $1 \leq p \leq 2$,

$$
\|B(f, g)\|_{L^{p}\left(\mathbb{R}^{d}\right)} \leq\|f\|_{L^{2}\left(\mathbb{R}^{d}\right)} \cdot\|g\|_{L^{2}\left(\mathbb{R}^{d}\right)} \cdot\left(\int|\widehat{K}(\xi,-\xi)|^{p^{\prime}} d \xi\right)^{\frac{1}{p^{\prime}}} .
$$


While Theorem 3.1 was useful in establishing geometric and combinatorial estimates, a weakness that limited wider applications was the positivity assumption on the kernel. This prevents one from exploiting, e.g., Fourier decay information by using analytic interpolation or Littlewood-Paley type decompositions. In the present work, Theorems 1.3 and Corollary 1.4 resolve this problem to a significant extent, opening the door to a variety of applications illustrated in this section.

3.1. $L^{p}$-improving measures. A finite measure $\mu$ on $\mathbb{R}^{d}$ is said to be $L^{p}$-improving if

$$
\|f * \mu\|_{L^{q}\left(\mathbb{R}^{d}\right)} \leq C_{p, q}\|f\|_{L^{p}\left(\mathbb{R}^{d}\right)} \text { for some } q>p .
$$

There has been much interest in determining the pairs $(p, q)$ for which (3.1) holds. Two representative results in this area are due to Strichartz [27] and Littman [20]; see Christ [7] for more recent work. The result of Littman and Strichartz says that if $\mu$ is the Lebesgue measure on the unit sphere in $\mathbb{R}^{d}, d \geq 2$, then (3.1) holds if and only if $(1 / p, 1 / q)$ is contained in the closed triangle with endpoints $(0,0),(1,1)$ and $\left(\frac{d}{d+1}, \frac{1}{d+1}\right)$.

In the multilinear setting, the notion of $L^{p}$-improving may be expressed in the following way, indicating an improvement over the Hölder inequality exponents governing pointwise multiplication.

Definition 3.2. Let $B\left(f_{1}, \ldots, f_{m}\right)$ be an $m$-linear operator. We say that $B$ is $L^{p}$ improving if there exist $p_{1}, \ldots, p_{m}, r>0$ with

$$
\frac{1}{p_{1}}+\cdots+\frac{1}{p_{m}}>\frac{1}{r}
$$

such that

$$
B: L^{p_{1}}\left(\mathbb{R}^{d}\right) \times \cdots \times L^{p_{m}}\left(\mathbb{R}^{d}\right) \rightarrow L^{r}\left(\mathbb{R}^{d}\right) .
$$

The following result is a simple adaptation of Corollary 1.4.

Theorem 3.3. Suppose that $\nu(\vec{u})$ is a smooth multiple of surface measure on a compact, codimension l submanifold of $\mathbb{R}^{m d}$. For $j \geq 0$, let $\nu_{j}$ be the $j$ th Littlewood-Paley component of $\nu$, and suppose that, for some $\gamma>0$,

$$
\left\|\nu_{j}\right\|_{\Lambda_{2, \infty}^{H_{0}}} \lesssim 2^{-j \gamma}
$$

Define

$$
B_{\nu}\left(f_{1}, \ldots, f_{m}\right)(x):=\int_{\mathbb{R}^{m d}} f_{1}\left(x-u^{1}\right) \cdots f_{m}\left(x-u^{m}\right) d \nu(\vec{u})
$$

Then

$$
\left\|B_{\nu}\left(f_{1}, \ldots, f_{m}\right)\right\|_{L_{\gamma}^{2}\left(\mathbb{R}^{d}\right)} \lesssim \prod_{j=1}^{m}\left\|f_{j}\right\|_{L^{2}\left(\mathbb{R}^{d}\right)}
$$

and

$$
\left\|B_{\nu}\left(f_{1}, \ldots, f_{m}\right)\right\|_{L^{p^{\prime}\left(\mathbb{R}^{d}\right)}} \lesssim \prod_{j=1}^{m}\left\|f_{j}\right\|_{L^{p}\left(\mathbb{R}^{d}\right)} \text { for } p>\frac{2(l+\gamma)}{l+2 \gamma}
$$


Remark 3.4. It is not difficult to check that if $|\widehat{\nu}(\vec{\xi})| \leq C(1+|\vec{\xi}|)^{-\alpha}$, then $(3.2)$ holds with the exponent $\gamma=\alpha-\frac{(m-1) d}{2}$.

Corollary 3.5. Let $\nu$ be surface measure on the sphere $\mathbb{S}^{m d-1}=\left\{\vec{u} \in \mathbb{R}^{m d}:\left|u^{1}\right|^{2}+\right.$ $\left.\cdots+\left|u^{m}\right|^{2}=1\right\}$. Then

$$
B_{\nu}: L^{p}\left(\mathbb{R}^{d}\right) \times \cdots \times L^{p}\left(\mathbb{R}^{d}\right) \rightarrow L^{p^{\prime}}\left(\mathbb{R}^{d}\right) \text { for } \frac{d+1}{d}<p \leq 2 .
$$

Corollary 3.5 follows from Theorem 3.3, since the codimension $l=1$ and the estimate (3.2) holds with $\gamma=\frac{m d-1}{2}-\frac{d}{2}$, using the Remark 3.4 and the well-known decay (see, e.g., [25]) that, if $\sigma$ denotes surface measure on the sphere in $\mathbb{R}^{n}$, $n \geq 2$, then

$$
|\widehat{\sigma}(\xi)| \leq C(1+|\xi|)^{-\frac{n-1}{2}} .
$$

One can in fact improve Corollary 3.5 to obtain the endpoint $p=\frac{d+1}{d}$ as follows. Define

$$
B_{\nu}^{z}\left(f_{1}, \ldots, f_{m}\right)(x):=\int f_{1}\left(x-u^{1}\right) \cdots f_{m}\left(x-u^{m}\right) d \nu^{z}(\vec{u}),
$$

where

$$
\nu^{z}(\vec{u})=\frac{1}{\Gamma(z)}\left(1-|\vec{u}|^{2}\right)_{+}^{z-1} .
$$

When $\operatorname{Re}(z)=-\frac{d-1}{2}, B_{\nu}^{z}: L^{2}\left(\mathbb{R}^{d}\right) \times \cdots \times L^{2}\left(\mathbb{R}^{d}\right) \rightarrow L^{2}\left(\mathbb{R}^{d}\right)$ via a direct appeal to Corollary 1.4 and the observation above that we may take all the way up to $\gamma=\frac{(m-1) d-1}{2}$ in $(3.2)$. When $\operatorname{Re}(z)=1, B_{\nu}: L^{1}\left(\mathbb{R}^{d}\right) \times \cdots \times L^{1}\left(\mathbb{R}^{d}\right) \rightarrow L^{\infty}\left(\mathbb{R}^{d}\right)$ trivially since the kernel becomes bounded. Stein's analytic interpolation theorem then yields the conclusion of Corollary 3.5 without the loss of the endpoint.

This estimate is basically sharp, as can be seen by the following adaptation of the usual linear sharpness example which we illustrate in the bilinear setting. Let $f$ be the characteristic function of the unit ball and let $g$ be the characteristic function of the ball of radius $\delta$. Then $\|g\|_{p} \approx \delta^{\frac{d}{p}}$ and $B_{\nu}(f, g) \approx \delta^{d-1}$ on the annulus of thickness $\approx \delta$ and radius $\approx 1$. Sharpness of the estimate above, up to the endpoint, follows.

In the bilinear case, if $\left\{\left(u^{1}, u^{2}\right):\left|u^{1}\right|^{2}+\left|u^{2}\right|^{2}=1\right\}$ is replaced by $\left\{\left(u^{1}, u^{2}\right): u^{1} \cdot u^{2}=\right.$ $t\}$ with $t \neq 0$ fixed, one obtains the same exponents as in Corollary 3.5. This surface arose in [11] in connection with the study of areas of triangles determined by fractal subsets of Euclidean space.

3.2. Proof of Theorem 3.3. The estimate (3.3) follows from Theorem 1.3 and (3.2). In fact, since $B_{\nu}\left(f_{1}, \ldots, f_{m}\right)=\left.\left(f_{1} \otimes \cdots \otimes f_{m}\right) * \nu\right|_{H_{0}}$, we have $\left(I-\Delta_{x}\right)^{\gamma / 2} B_{\nu}\left(f_{1}\right.$, $\left.\ldots, f_{m}\right)=\left.\left(f_{1} \otimes \cdots \otimes f_{m}\right) * V\right|_{H_{0}}$, where $\widehat{V}\left(\xi^{1}, \ldots, \xi^{m}\right)=\left(1+\left|\xi^{1}+\cdots+\xi^{m}\right|^{2}\right)^{\gamma / 2} \widehat{\nu}$. From (3.2), we see that $\|V\|_{\Lambda_{2, \infty}^{H_{0}}} \lesssim 1$, and hence applying Theorem 1.3 with $r=p=$ $2, q=\infty$ yields $(3.3)$.

To prove (3.4), define as above the $j$ th Littlewood-Paley piece $\nu_{j}$ of $\nu, 0 \leq j<\infty$, and set

$$
B^{j}\left(f_{1}, \ldots, f_{m}\right)(x)=\int \cdots \int f_{1}\left(x-u^{1}\right) \cdots f_{m}\left(x-u^{m}\right) \nu_{j}(\vec{u}) d \vec{u}
$$


It follows from (3.3) that

$$
B^{j}: L^{2}\left(\mathbb{R}^{d}\right) \times \cdots \times L^{2}\left(\mathbb{R}^{d}\right) \rightarrow L^{2}\left(\mathbb{R}^{d}\right) \text { with norm } 2^{-j \gamma} .
$$

On the other hand,

$$
\left\|\left.B^{j}\left(f_{1}, \ldots, f_{m}\right)\right|_{L^{\infty}\left(\mathbb{R}^{d}\right)} \leq\right\| f_{1}\left\|_{L^{1}\left(\mathbb{R}^{d}\right)} \cdots\right\| f_{m}\left\|_{L^{1}\left(\mathbb{R}^{d}\right)} \cdot\right\| \nu_{j} \|_{L^{\infty}\left(\mathbb{R}^{m d}\right)} .
$$

By a direct calculation (see, e.g., [31]), using the codimension $l$ of the support of $\nu$,

$$
\left\|\nu_{j}\right\|_{L^{\infty}\left(\mathbb{R}^{m d}\right)} \leq C 2^{l j} .
$$

The Riesz-Thorin interpolation theorem then yields (3.4).

Corollary 3.6. Let $\nu$ be surface measure on $S^{d-1} \times \cdots \times S^{d-1} \subset \mathbb{R}^{m d}$. Then

$$
B_{\nu}: L^{p}\left(\mathbb{R}^{d}\right) \times \cdots \times L^{p}\left(\mathbb{R}^{d}\right) \rightarrow L^{p^{\prime}}\left(\mathbb{R}^{d}\right) \text { for } \frac{\left(m^{2}-2 m+2\right) d+m^{2}}{\left(m^{2}-2 m+2\right) d} \leq p \leq 2 .
$$

To prove this, define $\nu^{z}(\vec{u})=\prod_{j=1}^{m} \frac{1}{\Gamma(z)}\left(1-\left|u^{j}\right|^{2}\right)_{+}^{z-1}$ and proceed as in the proof of Corollary 3.5.

3.3. Bilinear analogues of Stein's spherical maximal theorem. The classical (linear) spherical maximal operator is given by

$$
\mathcal{M} f(x)=\sup _{t>0}\left|\int_{S^{d-1}} f(x-t y) d \sigma(y)\right|,
$$

where $\sigma$ is the Lebesgue measure on the unit sphere in $\mathbb{R}^{d}, d \geq 2$. It was established by Stein [24] in three dimensions and higher, and by Bourgain [3] in two dimensions that

$$
\mathcal{M}: L^{p}\left(\mathbb{R}^{d}\right) \rightarrow L^{p}\left(\mathbb{R}^{d}\right) \text { for } p>\frac{d}{d-1} .
$$

The result is best possible, as can be shown by taking

$$
f(x)=|x|^{-d+1} \log \left(\frac{1}{|x|}\right) \chi_{B\left(\overrightarrow{0}, \frac{1}{2}\right)}(x) \text { with } t=|x| .
$$

The cornerstone of the general theory of maximal averages in dimension $d \geq 3$ is the following; cf. Stein [24], Greenleaf [14], Sogge and Stein [22], Bourgain [4], Carbery [6] and Cowling and Mauceri [9], Iosevich and Sawyer [17, 18].

Theorem 3.7. Let $\mu$ be a finite, compactly supported measure on $\mathbb{R}^{d}$, such that, for some $\epsilon>0,|\widehat{\mu}(\xi)| \leq C(1+|\xi|)^{-\frac{1}{2}-\epsilon}$. Define

$$
\mathcal{M}_{\mu} f(x)=\sup _{t>0}\left|\int f(x-t y) d \mu(y)\right| \text {. }
$$

Then, $\mathcal{M}_{\mu}: L^{2}\left(\mathbb{R}^{d}\right) \rightarrow L^{2}\left(\mathbb{R}^{d}\right)$.

The techniques introduced above yield multilinear analogues of these maximal theorems. For simplicity, we restrict ourselves to the bilinear case. Given a finite Borel measure $\nu$ on $\mathbb{R}^{2 d}$, define the bilinear averaging operators,

$$
B_{t}(f, g)(x)=\iint f(x-t u) g(x-t v) d \nu(u, v), 0<t<\infty
$$

and the associated maximal operator, $\mathcal{B}_{\nu}(f, g)(x)=\sup _{t>0}\left|B_{t}(f, g)(x)\right|$. The following bilinear analogue of Theorem 3.7 is an immediate restatement of Corollary 1.10. 
Theorem 3.8. Suppose that, for all $t>0$ and some $\epsilon>0$,

$$
\sup _{\xi \in \mathbb{R}^{d}}\left(\int_{2^{j} \leq \sqrt{|t \xi|^{2}+|t \eta|^{2}} \leq 2^{j+1}}\left|\widehat{\nu}\left(\frac{t \xi+t \eta}{2}, \frac{t \xi-t \eta}{2}\right)\right|^{2} d \eta\right)^{1 / 2} \leq C 2^{-j\left(\frac{1}{2}+\epsilon\right)},
$$

and the same estimate holds for $|\nabla \hat{\nu}|$. Then $\mathcal{B}_{\nu}: L^{2}\left(\mathbb{R}^{d}\right) \times L^{2}\left(\mathbb{R}^{d}\right) \rightarrow L^{2}\left(\mathbb{R}^{d}\right)$.

Using additional geometric information on the support of $\nu$, one can improve the exponents in Theorem 3.8 as follows.

Corollary 3.9. Suppose that $\nu$ is supported on a smooth compact submanifold of $\mathbb{R}^{d} \times \mathbb{R}^{d}$ of codimension $l$. Suppose that, for all $t>0$ and some $\gamma>\frac{1}{2}$,

$$
\sup _{\xi \in \mathbb{R}^{d}}\left(\int_{2^{j} \leq \sqrt{|t \xi|^{2}+|t \eta|^{2}} \leq 2^{j+1}}\left|\widehat{\nu}\left(\frac{t \xi+t \eta}{2}, \frac{t \xi-t \eta}{2}\right)\right|^{2} d \eta\right)^{1 / 2} \leq C 2^{-j \gamma}, \quad j \geq 0
$$

and the same holds for the gradient. Then

$$
\mathcal{B}_{\nu}: L^{p}\left(\mathbb{R}^{d}\right) \times L^{p}\left(\mathbb{R}^{d}\right) \rightarrow L^{p^{\prime}}\left(\mathbb{R}^{d}\right) \text { with } p>\frac{2 l+2 \gamma-1}{l+2 \gamma-1} .
$$

Remark 3.10. By a standard argument involving the approximation of an $L^{p}$ function by a continuous function plus a function with a small $L^{p}$ norm, Theorem 3.8 and Corollary 3.9 yield differentiation theorems. For example, Corollary 3.9 implies that if $f, g \in L^{p}\left(\mathbb{R}^{d}\right)$ with $p$ as in $(3.9)$, then

$$
\lim _{t \rightarrow 0} \iint f(x-t u) g(x-t v) d \nu(u, v)=f(x) g(x) \text { a.e. }
$$

Example 3.11. Let $\nu$ be the Lebesgue measure on the sphere $\left\{(u, v) \in \mathbb{R}^{d} \times \mathbb{R}^{d}\right.$ : $\left.|u|^{2}+|v|^{2}=1\right\}, d \geq 2$. Then

$$
\mathcal{B}_{\nu}: L^{p}\left(\mathbb{R}^{d}\right) \times L^{p}\left(\mathbb{R}^{d}\right) \rightarrow L^{p^{\prime}}\left(\mathbb{R}^{d}\right) \text { for } p>\frac{d}{d-1} .
$$

To see that the exponent $\frac{d}{d-1}$ is optimal, take $g \equiv 1$. What we get is essentially the maximal averaging operator of $f$ over the sphere in $\mathbb{R}^{d}$ of radius $1-|v|^{2}$. The needed restriction follows from Stein's sharpness example for the linear spherical maximal operator, namely, $f(x)=|x|^{-d+1} \log \left(\frac{1}{|x|}\right) \chi_{B\left(\overrightarrow{0}, \frac{1}{2}\right)}(x)$.

3.4. Proof of Corollary 3.9. By a direct calculation,

$$
\left|B_{t}^{j}(f, g)(x)\right| \leq\|f\|_{1} \cdot\|g\|_{1} \cdot\left\|K^{j}\right\|_{\infty} \leq C\|f\|_{1} \cdot\|g\|_{1} \cdot 2^{j l} .
$$

The proof of Theorem 3.8 above gives

$$
\mathcal{B}^{j}: L^{2}\left(\mathbb{R}^{d}\right) \times L^{2}\left(\mathbb{R}^{d}\right) \rightarrow L^{2}\left(\mathbb{R}^{d}\right) \text { with norm } \approx 2^{-j\left(\gamma-\frac{1}{2}\right)},
$$

and using Riesz-Thorin again yields Corollary 3.9.

\section{Applications to PDE}

We now show that the results established so far can be used to obtain a variety of estimates related to Sobolev spaces and PDE. 
4.1. Sobolev traces on subspaces. The classical Sobolev trace inequality from $\mathbb{R}^{n}$ to $\mathbb{R}^{n-1}$ (see, e.g., [29, Proposition 1.6]) says that if the restriction (or trace) map $\tau$ is defined by $\tau(u)=f$, where $f\left(x^{\prime}\right)=u\left(0, x^{\prime}\right), x=\left(x_{1}, x^{\prime}\right), x^{\prime}=\left(x_{2}, \ldots, x_{n}\right)$, then

$$
\|\tau(u)\|_{L_{s-\frac{1}{2}}^{2}\left(\mathbb{R}^{n-1}\right)} \leq C_{s}\|u\|_{L_{s}^{2}\left(\mathbb{R}^{n}\right)}, \quad s>\frac{1}{2} .
$$

Iterating (4.1) $n-k$ times yields, for any $k$-dimensional subspace $H \subset \mathbb{R}^{n}$,

$$
\|\tau(u)\|_{L_{s-\frac{n-k}{2}}^{2}(H)} \leq C_{s}^{\prime}\|u\|_{L_{s}^{2}\left(\mathbb{R}^{2 d}\right)}, \quad s>\frac{n-k}{2} .
$$

We shall use Theorem 1.3 to prove an endpoint version of (4.2) with the best constant, and then give some applications to PDE.

Theorem 4.1. If $H \subset \mathbb{R}^{n}$ is an affine subspace with $\operatorname{dim}(H)=k$, and $s>\frac{n-k}{2}$, then

$$
\left\|\left.u\right|_{H}\right\|_{L^{2}(H)} \leq C_{s, H}\|u\|_{L_{s}^{2}\left(\mathbb{R}^{n}\right)}, \quad \forall u \in \mathcal{S}\left(\mathbb{R}^{n}\right) .
$$

Furthermore, the optimal constant is

$$
C_{s, n-k}=\left\|\left(1+|\xi|^{2}\right)^{-\frac{s}{2}}\right\|_{\Lambda_{2, \infty}^{H}}=\sqrt{\left|\mathbb{S}^{n-k-1}\right| \cdot \int_{0}^{\infty}\left(1+r^{2}\right)^{-s} r^{n-k-1} d r} .
$$

Remark 4.2. Theorem 4.1 is result is an endpoint version of (4.2) in the sense that we take $L^{2}(H)$ on the left-hand side instead of $L_{s-\frac{n-k}{2}}^{2}(H)$ for $s>\frac{n-k}{2}$. One cannot pin the endpoint on both sides, as boundedness $\tau: L_{\frac{d}{2}}^{2}\left(\mathbb{R}^{2 d}\right) \rightarrow L^{2}(H)$ does not hold. Clearly, if $C_{s, n-k}$ is as in (4.4) then $\lim _{s \backslash \frac{n-k}{2}} C_{s, n-k}=\infty$.

Proof of Theorem 4.1. As in the proof of Theorem 1.3, by translation- and rotationinvariance of the function spaces involved, we can assume that $H=\left\{\left(x^{\prime}, x^{\prime \prime}\right) \in\right.$ $\left.\mathbb{R}^{k} \times \mathbb{R}^{n-k} \mid x^{\prime \prime}=0\right\}$, so that $H^{*} \simeq \mathbb{R}_{\xi^{\prime}}^{k}$ and $H_{\xi^{\prime}}^{\perp}=\left\{\left(\xi^{\prime}, \xi^{\prime \prime}\right) \mid \xi^{\prime \prime} \in \mathbb{R}^{n-k}\right\}$. One can rewrite $(4.3)$ in the form

$$
\left\|(-\triangle)^{-\frac{s}{2}} u\right\|_{L^{2}(H)} \leq C_{s}\|u\|_{L^{2}\left(\mathbb{R}^{2 d}\right)}, \quad s>\frac{d}{2} .
$$

The left-hand side can be rewritten as

$$
\left\|u * G_{s}\right\|_{L^{2}(H)} \text { with } \widehat{G}_{s}\left(\xi^{\prime}, \xi^{\prime \prime}\right)=\left(1+\left|\xi^{\prime}\right|^{2}+\left|\xi^{\prime \prime}\right|^{2}\right)^{-\frac{s}{2}} .
$$

By Theorem 1.3 we conclude that (4.3) holds, with optimal constant

$$
C_{s, n-k}=\left\|\left(1+|\xi|^{2}+\left|\xi^{\prime \prime}\right|^{2}\right)^{-\frac{s}{2}}\right\|_{\Lambda_{2, \infty}^{H}} .
$$

The $\sup _{\xi^{\prime}}$ in the norm on the right is attained at $\xi^{\prime}=0$, yielding

$$
C_{s, n-k}^{2}=\left|\mathbb{S}^{n-k-1}\right| \cdot \int_{0}^{\infty}\left(1+r^{2}\right)^{-s} r^{n-k-1} d r<\infty, \quad s>\frac{n-k}{2},
$$

completing the proof of (4.3) with constant (4.4).

We also can give a simple proof of the following product Sobolev estimate of Tambaca [28]: 
Theorem 4.3. Let $u, v \in \mathcal{S}\left(\mathbb{R}^{d}\right)$. Suppose that $\gamma=r+s-\frac{d}{2}$ and $r, s \geq 0$. Then

$$
\|u v\|_{L_{\gamma}^{2}\left(\mathbb{R}^{d}\right)} \leq C\|u\|_{L_{r}^{2}\left(\mathbb{R}^{d}\right)} \cdot\|v\|_{L_{s}^{2}\left(\mathbb{R}^{d}\right)} \cdot
$$

Remark 4.4. Unfortunately, we are not able to obtain the best constant in (4.5), since we cannot be certain that the optimizing function for the inequality (1.5) is realizable as a product function.

Proof of Theorem 4.3. We make use of the bilinear convolution estimates from Corollary 1.4. Let

$$
f=(-\triangle)^{\frac{s}{2}} u \text { and } g=(-\triangle)^{\frac{r}{2}} .
$$

We can rewrite $(4.5)$ in the form

$$
\|u v\|_{L_{\gamma}^{2}\left(\mathbb{R}^{d}\right)} \leq C\|f\|_{L^{2}\left(\mathbb{R}^{d}\right)} \cdot\|g\|_{L^{2}\left(\mathbb{R}^{d}\right)} .
$$

Let

$$
B(f, g)(x)=c_{r, s} \iint f(x-u) g(x-v)|u|^{-d+r}|v|^{-d+s} d u d v
$$

and observe that, with the appropriate choice of $c_{r, s}$, one has $B(f, g)=u v$. Applying Corollary 1.4, we see that

$$
\|u v\|_{L^{2}\left(\mathbb{R}^{d}\right)} \leq\|f\|_{L^{2}\left(\mathbb{R}^{d}\right)} \cdot\|g\|_{L^{2}\left(\mathbb{R}^{d}\right)} \cdot \sup _{\xi \in \mathbb{R}^{d}}\left(\int\left|\widehat{K}\left(\frac{\xi+\eta}{2}, \frac{\xi-\eta}{2}\right)\right|^{2} d \eta\right)^{1 / 2},
$$

where $\widehat{K}(\xi, \eta)=|\xi|^{-r}|\eta|^{-s}$. Since

$$
\left(\int_{|\xi|+|\eta| \approx 2^{j}}\left|\widehat{K}\left(\frac{\xi+\eta}{2}, \frac{\xi-\eta}{2}\right)\right|^{2} d \eta\right)^{\frac{1}{2}} \approx 2^{-j\left(r+s-\frac{d}{2}\right)},
$$

the result follows.

We now give some applications of Theorems 4.1 and 4.3 to a priori estimates in PDE.

4.2. The heat equation: restriction of solutions to subspaces. Consider the heat equation on $\mathbb{R}^{n} \times \mathbb{R}_{+}$,

$$
\frac{\partial u}{\partial t}=\triangle u,(x, t) \in \mathbb{R}^{n} \times \mathbb{R}_{+}, \quad u(x, 0)=F(x) \in L^{2}\left(\mathbb{R}^{n}\right),
$$

with solution given by $u(x, t)=\left(F * \Phi_{t}\right)(x)$, where

$$
\Phi_{t}(x)=(4 \pi t)^{-\frac{n}{2}} e^{-\frac{|x|^{2}}{4 t}} .
$$

We have the following result concerning the restriction of the heat semi-group to affine subspaces.

Theorem 4.5. Suppose that $u$ is the solution to (4.6). Then, for any $k$-dimensional affine subspace $H \subset \mathbb{R}^{n}$,

$$
\left\|\left.u(\cdot, t)\right|_{H}\right\|_{L^{2}(H)} \leq(4 \pi t)^{-\frac{n-k}{4}} \cdot\|F\|_{L^{2}\left(\mathbb{R}^{n}\right)},
$$

and the constant $(4 \pi t)^{-\frac{n-k}{4}}$ is optimal. 
In other words, the initial data $F$ might blowup along $H$ for $t$ small, resulting in the solution $u$ being large along $H$ for small values of $t$, but after the fixed time $T=\frac{1}{4 \pi}$, independent of $F$, the $L^{2}(H)$ norm of the solution drops below the $L^{2}\left(\mathbb{R}^{n}\right)$ norm of $F$.

Proof of Theorem 4.5. By invariance of (4.6) under the Euclidian motion group, as in the proof of Thm. 4.1H may be taken to be $H=\left\{\left(x^{\prime}, 0\right) \in \mathbb{R}^{n} \mid x^{\prime} \in \mathbb{R}^{k}\right\}$. Since $\widehat{\Phi}_{t}(\xi)=e^{-4 \pi^{2} t|\xi|^{2}}=e^{-4 \pi^{2} t\left(\left|\xi^{\prime}\right|^{2}+\left|\xi^{\prime \prime}\right|^{2}\right)}$, one has

$$
\begin{aligned}
\left\|\Phi_{t}\right\|_{\Lambda_{2, \infty}^{H}} & =\sup _{\xi^{\prime} \in \mathbb{R}^{k}} e^{-2 \pi^{2} t\left|\xi^{\prime}\right|^{2}}\left(\int_{\mathbb{R}^{n-k}} e^{-4 \pi^{2} t\left|\xi^{\prime \prime}\right|^{2}} d \xi^{\prime \prime}\right)^{1 / 2} \\
& =\left(\int_{\mathbb{R}^{n-k}} e^{-4 \pi^{2} t\left|\xi^{\prime \prime}\right|^{2}} d \xi^{\prime \prime}\right)^{1 / 2} \\
& =\left((2 \pi \sqrt{t})^{-\frac{n-k}{2}} \int_{\mathbb{R}^{n-k}} e^{-\left|z^{\prime \prime}\right|^{2}} d z^{\prime \prime}\right)^{\frac{1}{2}}=(4 \pi t)^{-\frac{n-k}{4}} .
\end{aligned}
$$

4.3. The wave equation: restriction to subspaces. We now consider solutions to the Cauchy problem for the wave equation,

$$
u_{t t}=\triangle u,(x, t) \in \mathbb{R}^{n} \times \mathbb{R}_{+}, u(x, 0)=0, u_{t}(x, 0)=F(x, y) \in L^{2}\left(\mathbb{R}^{n}\right) .
$$

As is well-known, the map $F(x) \longrightarrow u(x, t)$ is bounded between a variety of function spaces; see, e.g., $[23,25]$ and references therein. We show here that for fixed $t \neq 0$ the solution $u(x, t)$ restricts in a natural way to any $k$-plane.

By the standard integral representation of the solution to (4.7),

$$
u(x, t)=F * K_{t}(x):=c_{n}\left(\frac{1}{t} \frac{d}{d t}\right)^{\frac{n-3}{2}}\left\{t^{n-2} A_{t} F(x, y)\right\},
$$

with, as before,

$$
A_{t} F(x, y)=\int_{\mathbb{S}^{n-1}} F(x-t u) d \sigma(u),
$$

where $d \sigma$ is the surface measure on the unit sphere in $\mathbb{R}^{n}$ and $K_{t}$ is the (fixed time) fundamental solution. The following is an immediate consequence of Theorem 1.3.

Theorem 4.6. Let $H$ be a $k$-dimensional affine subspace of $\mathbb{R}^{n}$. If $s<1-\frac{n-k}{2}$, then, for all $t>0$,

$$
\left\|\left.\left(I-\triangle_{x}\right)^{\frac{s}{2}} u(\cdot, t)\right|_{H}\right\|_{L^{2}(H)} \leq C\|F\|_{L^{2}\left(\mathbb{R}^{n}\right)},
$$

with the optimal constant given by

$$
C_{s, n-k, t}=\left\|\left(I-\triangle_{x}\right)^{\frac{s}{2}} K_{t}\right\|_{\Lambda_{2, \infty}^{H}\left(\mathbb{R}^{n}\right)} .
$$

Proof. Since $\left|\left(\left(I-\triangle_{x}\right)^{s} K_{t}\right) \hat{(\xi)}\right| \lesssim(1+|\xi|)^{s-1}$, after putting $H$ into the same form $\left\{\left(x^{\prime}, 0\right) \in \mathbb{R}^{n} \mid x^{\prime} \in \mathbb{R}^{k}\right\}$ as used above, we see that, for $s<1$,

$$
\left\|\left(I-\triangle_{x}\right)^{s} K_{t}\right\|_{\Lambda_{2, \infty}^{H}}<\infty, \quad \text { iff } \quad \int_{\mathbb{R}^{n-k}}\left(1+\left|\xi^{\prime \prime}\right|^{2}\right)^{2 s-2} d \xi^{\prime \prime}<\infty,
$$

which holds iff $2 s-2<-(n-k)$, i.e., $s<1-\frac{n-k}{2}$. 
4.4. The wave equation: product of solutions. Consider solutions $u$ and $v$ on $\mathbb{R}^{3+1}$ to

$u_{t t}=\triangle u, u(x, 0)=0, u_{t}(x, 0)=f(x), \quad$ and $\quad v_{t t}=\triangle v, v(x, 0)=0, v_{t}(x, 0)=g(x)$.

We have the following estimate for products of solutions to these; see [10] for similar results with an additional average in time.

Theorem 4.7. With the notation above, for any fixed $t$,

$$
\begin{gathered}
\|u(\cdot, t) v(\cdot, t)\|_{L^{3}\left(\mathbb{R}^{3}\right)} \leq C\|u(\cdot, t) v(\cdot, t)\|_{L_{\frac{1}{2}}^{2}\left(\mathbb{R}^{3}\right)} \leq C^{\prime}\|f\|_{L^{2}\left(\mathbb{R}^{3}\right)} \cdot\|g\|_{L^{2}\left(\mathbb{R}^{3}\right)}, \\
\|u(\cdot, t) v(\cdot, t)\|_{L^{p^{\prime}\left(\mathbb{R}^{3}\right)}} \leq C_{p, t}\|f\|_{L^{p}\left(\mathbb{R}^{3}\right)} \cdot\|g\|_{L^{p}\left(\mathbb{R}^{3}\right)} \text { for } \frac{5}{3}<p \leq 2 .
\end{gathered}
$$

Remark 4.8. One then obtains $L^{p} \times L^{p} \rightarrow L^{q}$ estimates for $\left(\frac{1}{p}, \frac{1}{q}\right)$ in a nonclosed triangle with vertices at $\left(\frac{1}{2}, \frac{1}{2}\right),\left(\frac{1}{2}, \frac{1}{3}\right)$ and $\left(\frac{3}{5}, \frac{2}{5}\right)$.

To prove Theorem 4.7 , observe that

$$
u(x, t)=\frac{t}{4 \pi} \int_{S^{2}} f(x-t y) d \sigma(y),
$$

so that estimate (4.9) follows immediately from Corollary 3.6. On the other hand, (4.8) follows from Theorem 4.3. In fact, since $|\widehat{\sigma}(t \xi)| \leq C(1+t|\xi|)^{-1}$, it follows that

$$
\|u(\cdot, t)\|_{L_{1}^{2}\left(\mathbb{R}^{3}\right)} \leq C\|f\|_{L^{2}\left(\mathbb{R}^{3}\right)} \text { and }\|v(\cdot, t)\|_{L_{1}^{2}\left(\mathbb{R}^{3}\right)} \leq C\|g\|_{L^{2}\left(\mathbb{R}^{3}\right)},
$$

with $C$ independent of $t$. Let $r=s=1$, so that $\gamma=2-\frac{3}{2}=\frac{1}{2}$. Theorem 4.3, followed by (4.10), implies

$$
\|u(\cdot, t) v(\cdot, t)\|_{L_{\frac{1}{2}}^{2}\left(\mathbb{R}^{3}\right)} \leq C\|u\|_{L_{1}^{2}\left(\mathbb{R}^{3}\right)}\|v\|_{L_{1}^{2}\left(\mathbb{R}^{3}\right)} \leq C\|f\|_{L^{2}\left(\mathbb{R}^{3}\right)} \cdot\|g\|_{L^{2}\left(\mathbb{R}^{3}\right)} .
$$

One then applies Sobolev embedding in $\mathbb{R}^{3}$ to obtain the first inequality in (4.8).

\section{Bilinear oscillatory integral operators}

Let $\phi: \mathbb{R}^{d} \times \mathbb{R}^{d} \rightarrow \mathbb{R}$ be a smooth phase function and $\psi \in C_{0}^{\infty}\left(\mathbb{R}^{2 d}\right)$ a fixed amplitude. Define

$$
T_{\phi}^{\lambda} F(x, y)=\iint F(x-u, y-v) e^{2 \pi i \lambda \phi(u, v)} \psi(u, v) d u d v .
$$

If $\phi$ is nondegenerate, i.e., the determinant of the Hessian matrix of $\phi$ does not vanish on the support of $\psi$, then it is a result of Hörmander that

$$
T_{\phi}^{\lambda}: L^{2}\left(\mathbb{R}^{2 d}\right) \rightarrow L^{2}\left(\mathbb{R}^{2 d}\right) \text { with norm } \leq C \lambda^{-d} .
$$

See [25] and references therein for this and related results. What we describe above works equally well in any $\mathbb{R}^{n}$, but this formulation leads naturally to the context of trace inequalities, which we now present.

Definition 5.1. The bilinear surface associated with $\phi$ is

$$
S_{\phi}=\left\{\left(u, v, z, \delta_{z} \phi(u, v)\right):(u, v) \in U ; z \in Z\right\} \subset \mathbb{R}^{3 d+1},
$$

where $U:=\operatorname{supp}(\psi), Z=\pi_{1}(U)-\pi_{1}(U)$, with $\pi_{1}(u, v)=u$, and

$$
\delta_{z} \phi(u, v)=\phi(u, v)-\phi(u-z, v-z)
$$


Let $\sigma_{\phi}$ denote the induced surface measure on $S_{\phi}$. We shall see below that Theorem 1.3 implies the following result.

Theorem 5.2. Let $H$ be a d-dimensional plane in $\mathbb{R}^{2 d}$. Then

$$
\left\|\left.\left(T_{\phi}^{\lambda} F\right)\right|_{H}\right\|_{L^{2}(H)} \leq \sqrt{\sup _{\xi \in \mathbb{R}^{d}}\left|\widehat{\sigma}_{\phi}(0,0, \xi, \lambda)\right|} \cdot\|F\|_{L^{2}\left(\mathbb{R}^{2 d}\right)} .
$$

We now apply our results to bilinear oscillatory integral operators, defined by

$$
M_{\phi}^{\lambda}(f, g)(x)=\iint f(x-u) g(x-v) e^{2 \pi i \lambda \phi(u, v)} \psi(u, v) d u d v .
$$

We have the following bound on the $L^{2}$-norm of $M_{\phi}^{\lambda}$, which follows at once from Theorem 5.2.

Corollary 5.3. Let $M_{\phi}^{\lambda}$ be as above. Then

$$
\left\|M_{\phi}^{\lambda}(f, g)\right\|_{2} \leq \sqrt{\sup _{\xi \in \mathbb{R}^{d}}\left|\widehat{\sigma}_{\phi}(0,0, \xi, \lambda)\right|} \cdot\|f\|_{2}\|g\|_{2} .
$$

Corollary 5.4. Suppose that the determinant of the Hessian matrix of $\phi$ does not vanish on the support of $\psi$. Then

$$
\left\|M_{\phi}^{\lambda}(f, g)\right\|_{2} \leq C \lambda^{-\frac{d}{2}}\|f\|_{2}\|g\|_{2} .
$$

Remark 5.5. The power of $\lambda$ in the estimate (5.3) cannot, in general, be improved. This can be checked by taking $\phi(u, v)=|u|^{2}+|v|^{2}$ and running a simple scaling argument.

Example 5.6. Let $\phi(u, v)=u \cdot v$. Then the conditions of Corollary 5.4 are satisfied and the conclusion holds.

Proof of Theorem 5.2. By Theorem 1.3, we need to compute the square root of

$$
\begin{aligned}
\sup _{\xi \in \mathbb{R}^{d}} & \int\left|\iint e^{-2 \pi i\left(\frac{\xi-\eta}{2} \cdot u+\frac{\xi+\eta}{2} \cdot v+\lambda \phi(u, v)\right)} \psi(u, v) d u d v\right|^{2} d \eta \\
= & \sup _{\xi \in \mathbb{R}^{d}} \iint \ldots \int e^{-2 \pi i\left(\frac{\xi-\eta}{2} \cdot\left(u-u^{\prime}\right)+\frac{\xi+\eta}{2} \cdot\left(v-v^{\prime}\right)+\lambda\left(\phi(u, v)-\phi\left(u^{\prime}, v^{\prime}\right)\right)\right)} \\
& \times \psi(u, v) \psi\left(u^{\prime}, v^{\prime}\right) d u d v d u^{\prime} d v^{\prime} d \eta \\
= & \sup _{\xi \in \mathbb{R}^{d}} \iint \ldots \int e^{-2 \pi i\left(\frac{\xi}{2} \cdot\left(u-u^{\prime}\right)+\frac{\xi}{2} \cdot\left(v-v^{\prime}\right)+\lambda\left(\phi(u, v)-\phi\left(u^{\prime}, v^{\prime}\right)\right)\right)} \\
& \times \psi(u, v) \psi\left(u^{\prime}, v^{\prime}\right) \delta_{0}\left(\left(u-u^{\prime}\right)-\left(v-v^{\prime}\right)\right) d u d v d u^{\prime} d v^{\prime},
\end{aligned}
$$

since the integral with respect to $\eta$ in the previous line yields $\delta_{0}\left(\left(u-u^{\prime}\right)-\left(v-v^{\prime}\right)\right)$, where $\delta_{0}$ denotes the $\delta$-distribution at the origin. Making the change of variables $z=u-u^{\prime}, u=u, v=v$, we obtain

$$
\sup _{\xi \in \mathbb{R}^{d}} \iiint e^{-2 \pi i\left(\xi \cdot z+\lambda \delta_{z} \phi(u, v)\right)} \psi(u, v) \psi(u-z, v-z) d u d v d z=\sup _{\xi \in \mathbb{R}^{d}} \widehat{\sigma}_{\phi}(0,0, \xi, \lambda),
$$

and the proof is complete. 
Proof of Corollary 5.4. By Theorem 5.2, we must estimate

$$
\sup _{\xi \in \mathbb{R}^{d}}\left|\iiint e^{-2 \pi i\left(z \cdot \xi+\lambda \delta_{z} \phi(u, v)\right)} \psi(u, v) \psi(u-z, v-z) d u d v d z\right| .
$$

By the method of stationary phase (see, e.g., [25]), our claim would follow if we could show that the rank of the $3 d \times 3 d$ Hessian matrix of $\Phi(u, v, z)=\delta_{z} \phi(u, v)$ has rank $\geq 2 d$. Since the argument is local, we can use the Morse lemma and establish the result with

$$
\phi(u, v)=u_{1}^{2} \pm u_{2}^{2} \pm \cdots \pm u_{d}^{2} \pm v_{1}^{2} \pm v_{2}^{2} \pm \cdots \pm v_{d}^{2}
$$

for which

$$
\Phi(u, v, z)=\sum_{i=1}^{d} \pm\left(\left(z_{i}-u_{i}\right)^{2}-u_{i}^{2}\right)+\sum_{j=1}^{d} \pm\left(\left(z_{j}-v_{j}\right)^{2}-v_{j}^{2}\right),
$$

and Corollary 5.4 follows.

\section{Further thoughts and open problems}

The key idea of this paper is that estimates for restrictions of convolutions (Theorem 1.3) provide a mechanism to obtain multilinear operator bounds, in the same way as the classical Young's inequality yields estimates for linear operators. This approach does not rely on positivity, which was a drawback of the the technique in [11]. Despite the applications we have presented in this paper, serious issues remain, especially regarding the bounds for bilinear operators arising in the geometric context. In [11], $L^{2} \times L^{2} \rightarrow L^{1}$ bounds were established for bilinear operators, with applications to Erdős/Falconer problems in geometry. These bounds relied heavily on the positivity of the kernel, which created significant obstacles, but the bounds were established in the arguably more natural range of exponents. While the problem of positivity was largely resolved in the current paper, the range of boundedness is centered around $L^{2} \times L^{2} \rightarrow L^{2}$. It would be extremely interesting to reconcile the methods in this paper and [11] and come up with a unified set of bounds that do not rely on positivity of the kernel. We now state some concrete open problems.

Problem 6.1. It is proved in [11] that if $B(f, g)$ is defined as above, then for $1 \leq r \leq 2$ and $K$ a non-negative finite measure,

$$
\|B(f, g)\|_{L^{r}\left(\mathbb{R}^{d}\right)} \leq\|f\|_{L^{2}\left(\mathbb{R}^{d}\right)} \cdot\|g\|_{L^{2}\left(\mathbb{R}^{d}\right)} \cdot\left(\int|\widehat{K}(\xi,-\xi)|^{r^{\prime}} d \eta\right)^{1 / r^{\prime}} .
$$

On the other hand, Corollary 1.7 above shows that, if $2 \leq r \leq \infty$,

$$
\|B(f, g)\|_{L^{r}\left(\mathbb{R}^{d}\right)} \leq\|f\|_{L^{2}\left(\mathbb{R}^{d}\right)}
$$

$$
\cdot\|g\|_{L^{2}\left(\mathbb{R}^{d}\right)} \cdot\left[\int\left(\int\left|\widehat{K}\left(\frac{\xi-\eta}{2}, \frac{\xi+\eta}{2}\right)\right|^{2} d \eta\right)^{\frac{r}{r-2}} d \xi\right]^{r-2 / 2 r}
$$


The two estimates agree at $r=2$ and the positivity of $K$ is clearly irrelevant there. The question we ask is whether it is possible to reconcile (6.1) and (6.2) without assuming that $K$ is positive.

Our second problem attempts to further address the issue raised in Corollary 1.7.

Problem 6.2. It follows from (1.10) that

$$
\left(\int_{\mathbb{R}^{d}}|\widehat{F}(x, x)|^{2} d x\right)^{\frac{1}{2}} \leq\left(\int_{\mathbb{R}^{d}}\left[\int_{\mathbb{R}^{d}}\left|F\left(\frac{x-y}{2}, \frac{x+y}{2}\right)\right| d y\right]^{2} d x\right)^{1 / 2}
$$

At least in two dimensions, it would be interesting to generalize this estimate to a universal $L^{2}$-restriction theorem where the left-hand side is

$$
\left(\int|\widehat{F}(x, \phi(x))|^{2} d x\right)^{1 / 2},
$$

with $\phi$ a suitably regular function of $x$, and the right-hand side is a mixed norm depending on $\phi$. In the special case when $\phi(x)=x^{2}$, say, the right-hand side should be comparable to the $\|F\|_{L^{\frac{6}{5}}\left(\mathbb{R}^{2}\right)}$, consistent with the Stein-Tomas restriction theorem.

\section{Acknowledgments}

The first three authors were supported by the NSF grants DMS-0747656, DMS0853892 and DMS-1045404, resp.

\section{References}

[1] I. Bejenaru, S. Herr and D. Tataru, A convolution estimate for two-dimensional hypersurfaces, Rev. Mat. Iberoamericana 26 (2010), 707-728.

[2] J. Bennett, A. Carbery and J. Wright, A non-linear generalisation of the Loomis-Whitney inequality and applications, Math. Res. Lett. 12 (2005), 443-457.

[3] J. Bourgain, Averages in the plane over convex curves and maximal operators, J. Anal. Math. 47 (1986), 69-85.

[4] J. Bourgain, On high-dimensional maximal functions associated to convex bodies, Amer. J. Math. 108(6) (1986), 1467-1476.

[5] P. Brass, W. Moser and J. Pach, Research Problems in Discrete Geometry, Springer, New York, 2005.

[6] A. Carbery, An almost-orthogonality principle with applications to maximal functions associated to convex bodies, Bull. Amer. Math. Soc. (N.S.) 14(2) (1986), 269-273.

[7] M. Christ, Convolution, curvature, and combinatorics: a case study, Int. Math. Res. Not. 1998(19) (1998), 1033-1048.

[8] R. Coifman and Y. Meyer, Wavelets. Calderón-Zygmund and multilinear operators, transl. by D. Salinger, Cambridge Studies in Advanced Mathematics, 48, Cambridge University Press, Cambridge, 1997.

[9] M. Cowling and G. Mauceri, Inequalities for some maximal functions. II. Trans. Amer. Math. Soc. 296(1) (1986), 341-365.

[10] D. Foschi and S. Klainerman, Bilinear space-time estimates for homogeneous wave equations Ann. Sci. École Norm. Sup. (4) 33(2) (2000), 211-274.

[11] L. Grafakos, A. Greenleaf, A. Iosevich and E. Palsson, Multilinear generalized Radon transforms and point configurations Forum Math., to appear.

[12] L. Grafakos and N. Kalton, Some remarks on multilinear maps and interpolation Math. Ann. 319(1) (2001), 151-180.

[13] L. Grafakos and R. Torres, On multilinear singular integrals of Calderón-Zygmund type (Proc. 6th Int. Conf. on Harm), Anal. and PDE, Publ. Mat., Barcelona, 2002. 
[14] A. Greenleaf, Principal curvature and harmonic analysis, Indiana Math. J. 30 (1982), 519-537.

[15] A. Greenleaf and A. Iosevich, On triangles determined by subsets of the Euclidean plane, the associated bilinear operators and applications to discrete geometry, Anal. PDE 5(2) (2012), 397-409.

[16] P. Gressman, On multilinear determinant functionals, Proc. Amer. Math. Soc. 139 (2011), 2473-2484.

[17] A. Iosevich and E. Sawyer, Maximal averages over surfaces, Adv. Math. 132(1) (1997), 46-119.

[18] A. Iosevich and E. Sawyer, Three problems motivated by the average decay of the Fourier transform, Contemp. Math., 320, Amer. Math. Soc., 2003.

[19] C. Kenig and E. Stein, Multilinear estimates and fractional integration, Math. Res. Lett. 6 (1999), 1-15.

[20] W. Littman, Lp-Lq-estimates for singular integral operators arising from hyperbolic equations, 'Partial Differential Equations' (Proc. Symp. Pure Math., Vol. XXIII, University of California, Berkeley, 1971), 479-481, Amer. Math. Soc., Providence, RI, 1973.

[21] G. Mockenhaupt, A. Seeger and C.D. Sogge, Wave front sets, local smoothing and Bourgain's circular maximal theorem, Ann. Math. (2) 136 (1992), 207-218.

[22] C. Sogge and E. Stein, Averages of functions over hypersurfaces in $\mathbb{R}^{n}$. Invent. Math. 82(3) (1985), 543-556.

[23] C.D. Sogge, Fourier Integrals in Classical Analysis, Cambridge University Press, 1993.

[24] E.M. Stein, Maximal functions: spherical means, Proc. Natl. Acad. Sci. USA 73 (1976), 21742175 .

[25] E. M. Stein, Harmonic Analysis, Princeton University Press, 1993.

[26] B. Stovall, $L^{p}$ improving multilinear Radon-like transforms, Rev. Mat. Iberoamericana $\mathbf{2 7}$ (2011), 1059-1085.

[27] R. Strichartz, Convolutions with kernels having singularities on a sphere, Trans. Amer. Math. Soc. 148 (1970), 461-471.

[28] J. Tambaca, Estimates of the Sobolev norm of a product of two functions, J. Math. Anal. Appl. 255 (2001), 137-146.

[29] M. Taylor, Partial Differential Equations, 23 Springer, TAM 1996.

[30] C. Thiele, Multilinear singular integrals (Proc. 6th Intl. Conf. Harm. Anal. and PDE), Publ. Mat., Barcelona, 2002.

[31] T. Wolff, Lectures on Harmonic Analysis (I. Laba and C. Shubin, eds.), University Lecture Series, 29. Amer. Math. Soc., Providence, RI, 2003.

Department of Mathematics, University of Rochester, Rochester, NY 14627, USA

E-mail address: dangeba@math.rochester.edu, allan@math.rochester.edu, iosevich@math. rochester.edu, palsson@math.rochester.edu

Department of Mathematics and Statistics, McMaster University, Hamilton, ON L8S 4K1, CANADA

E-mail address: sawyer@mcmaster.ca 